The Supporting Information published June 29, 2006 contained an incorrect procedural step on page S6. The corrected version was published December 7, 2006.

\title{
Synthesis of $N$-Methoxy- $N$-methyl- $\beta$-enaminoketoesters: New Synthetic Precursors for the Regioselective Synthesis of Heterocyclic Compounds
}

\author{
Tobias Persson and John Nielsen
}

Department of Natural Sciences, Bioorganic section, The Royal Danish Agricultural and Veterinary University, DK-1871 Frederiksberg C, Denmark

$$
\text { jn@kvl.dk }
$$

\section{Supporting information.}

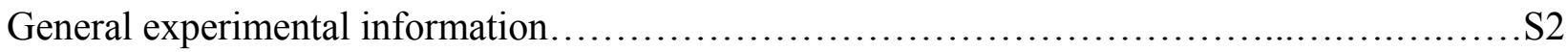

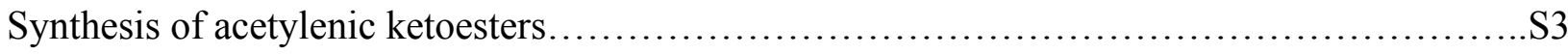

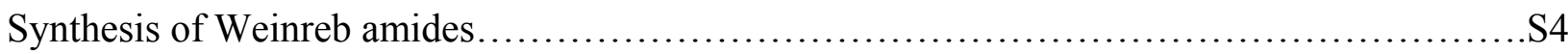

General procedure for the synthesis of $N$-methoxy- $N$-methyl- $\beta$-enaminoketoesters...........S6

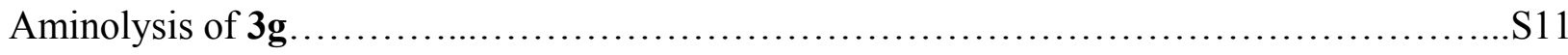

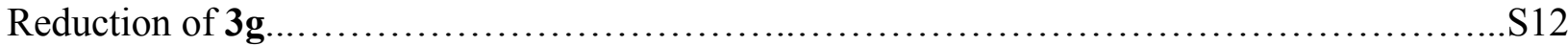

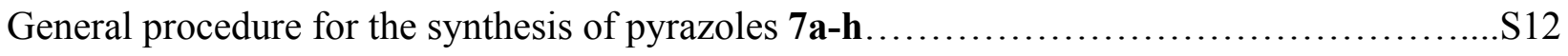

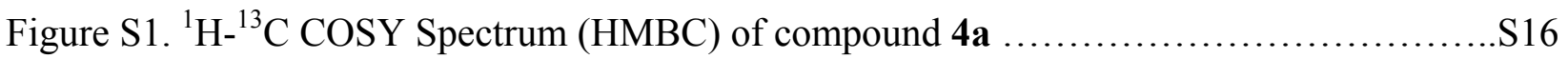

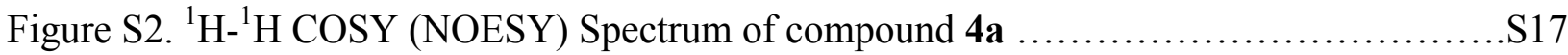

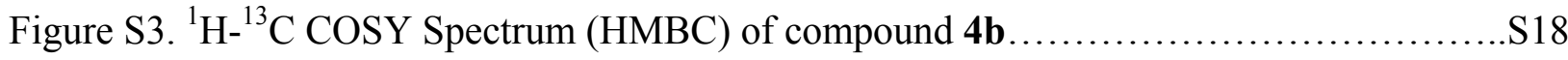

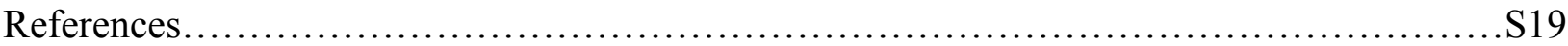




\section{General experimental information}

Commercially available reagents (Aldrich) were used without further purification unless otherwise noted. Solvents used for the synthesis were of analytical grade, dried over activated 4 $\AA$ molecular sieves when necessary (all solvents used under dry conditions had a water content $<$ $25 \mathrm{ppm}$ measured by Karl-Fischer titration). All reactions employing ethyl propynoate as starting material were performed under dry conditions in an atmosphere of argon (dried by passage through phosphorus pentoxide). MilliQ water was used for RP-HPLC. Analytical TLC was performed using pre-coated silica gel $60 \mathrm{~F}_{254}$ plates and visualized using either UV light, phosphomolybdic acid or potassium permanganate stain. Flash chromatography was performed automatically on a Biotage Quad 3+ Parallel Flash Purification ${ }^{\mathrm{TM}}$ system with pre-packed columns (KP-SIL, 32-63 $\mu \mathrm{m}, 60 \AA$ ). Reactions requiring microwave heating were performed in sealed pressure-rated reaction tubes on an Emrys ${ }^{\mathrm{TM}}$ Creator from Personal Chemistry. Corrected melting points were measured in open capillary tubes on a Gallenkamp electrothermal melting point apparatus (3g, 7a and $\mathbf{7 g}$ ) or on a Reichert melting point microscope, number N254-1R (2h, 2j, 3a, 3h, 7b, 7d, 7f and 7h). ${ }^{1} \mathrm{H}$ and ${ }^{13} \mathrm{C}$ NMR spectral data were recorded on a Bruker Avance 300 using the deuterated solvent as lock. Chemical shifts are reported in ppm relative to the residual solvent peak $\left({ }^{1} \mathrm{H}\right.$ NMR $)$ or the solvent peak $\left({ }^{13} \mathrm{C}\right.$ NMR $)$ as the internal standard. Accurate mass determinations were performed on a Micromass LCT apparatus equipped with an AP-ESI probe calibrated with Leu-Enkephalin $(556.2771 \mathrm{~g} / \mathrm{mol})$. HPLC was performed on a Waters 2525 system equipped with a Waters 2996 photodiode array detector and Xterra C18 Columns (anal.: $4.6 \times 100 \mathrm{~mm}, 5 \mu \mathrm{m}$; prep.: $19 \times 100 \mathrm{~mm}, 5 \mu \mathrm{m}$ ) with a flow of $1.0 \mathrm{~mL} / \mathrm{min}$. (anal.) or 15 $\mathrm{mL} /$ min. (prep.) $35 \% \rightarrow 25 \%$ A $1-7$ minutes $\left(\mathrm{A}: \mathrm{H}_{2} 0 ; \mathrm{B}: \mathrm{CH}_{3} \mathrm{CN}\right)$. Purity determinations of all 
substances stable in water were made by anal. HPLC (220 nm) and products of $>98 \%$ purity were considered homogeneous.

\section{Synthesis of acetylenic ketoesters}

\section{Ethyl 4-oxo-4-phenylbut-2-ynoate}

During 15 minutes a 1.0 M THF-solution of LHMDS (5.0 mL, 5.0 mmol) was added to a THFsolution $(5 \mathrm{~mL})$ of ethyl propynoate $(4.5 \mathrm{mmol}, 460 \mu \mathrm{L})$ cooled at $-78{ }^{\circ} \mathrm{C}$. The mixture was stirred for another half hour before addition of benzoyl chloride (590 $\mu \mathrm{L}, 5.0 \mathrm{mmol})$. After stirring for $3 \mathrm{~h}$ at the same temperature the reaction was quenched by addition of water $(25 \mathrm{~mL})$. The water phase was extracted with ether $(3 \times 25 \mathrm{~mL})$ and the combined organic phases dried $\left(\mathrm{MgSO}_{4}\right)$, filtered and evaporated to dryness. Flash chromatography (1:99 EtOAc/hexane) provided ethyl 4-oxo-4-phenyl-but-2-ynoate as an oil in 7\% (67 mg, $0.33 \mathrm{mmol})$ yield. ${ }^{1} \mathrm{H}$ NMR and ${ }^{13} \mathrm{C}$ NMR spectral data were in agreement with those previously reported. ${ }^{1}$

\section{Ethyl 4-oxo-pent-2-ynoate}

During 15 minutes a 1.0 M THF-solution of LHMDS (5.0 mL, 5.0 mmol) was added to a THFsolution $(5 \mathrm{~mL})$ of ethyl propynoate $(4.5 \mathrm{mmol}, 460 \mu \mathrm{L})$ cooled at $-78{ }^{\circ} \mathrm{C}$. The mixture was stirred for another half hour before transfer via cannula to a THF-solution $(10 \mathrm{~mL})$ of acetyl chloride $(2.4 \mathrm{~mL}, 22.5 \mathrm{mmol})$ cooled at $-78^{\circ} \mathrm{C}$. After stirring for $1 \mathrm{~h}$ at the same temperature the reaction was quenched by addition of water $(25 \mathrm{~mL})$. The water phase was extracted with ether $(3$ $\times 25 \mathrm{~mL})$ and the combined organic phases dried $\left(\mathrm{MgSO}_{4}\right)$, filtered and evaporated to dryness. Flash chromatography (EtOAc/hexane 1:99) provided ethyl 4-oxo-pent-2-ynoate as an oil in 9\% (58 mg, $0.41 \mathrm{mmol}$ ) yield. ${ }^{1} \mathrm{H}$ NMR and ${ }^{13} \mathrm{C}$ NMR spectral data were in agreement with those previously reported. ${ }^{2}$ 


\section{Synthesis of Weinreb amides according to Li et al. ${ }^{3}$}

$N$-Methoxy- $N$-methylpropionamide (2b)

Compound $\mathbf{2 b}$ was synthesized from propionyl chloride and isolated as a colorless oil in $51 \%$ yield (UV purity: 98\%) by distillation. ${ }^{1} \mathrm{H}$ NMR and ${ }^{13} \mathrm{C}$ NMR spectral data were in agreement with those previously reported. ${ }^{4}$

\section{$N$-Methoxy- $N$-methyl-2-phenylacetamide (2c)}

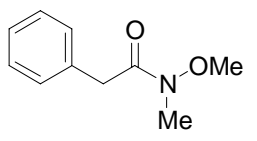

Compound 2c was synthesized from phenylacetyl chloride and isolated as a colorless oil in 65\% yield (UV purity: 98\%) after purification by flash chromatography (EtOAc/hexane 10:90): ${ }^{1} \mathrm{H}$ NMR and ${ }^{13} \mathrm{C}$ NMR spectral data were in agreement with those previously reported. ${ }^{5}$

\section{$N$-Methoxy- $N$-methyl-3-phenylpropionamide (2d)}

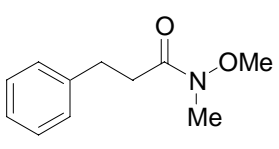

Compound 2d was synthesized from hydrocinnamoyl chloride and isolated as a colorless oil in 84\% yield (UV purity: 85\%) after purification by flash chromatography (EtOAc/hexane 10:90): ${ }^{1} \mathrm{H}$ NMR and ${ }^{13} \mathrm{C}$ NMR spectral data were in agreement with those previously reported. ${ }^{6}$

\section{$N$-Methoxy- $N$-methylisobutyroamide (2e)}

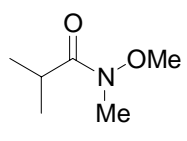

Compound 2e was synthesized from isobutyryl chloride and isolated as a colorless oil in 13\% yield (UV purity: 96\%) after purification by flash chromatography (EtOAc/heptane 10:90): ${ }^{1} \mathrm{H}$ NMR (300 MHz, $\left.\mathrm{CDCl}_{3}\right) \delta 3.69(\mathrm{~s}, 3 \mathrm{H}), 3.18(\mathrm{~s}, 3 \mathrm{H}), 2.96$ (heptet, $\left.{ }^{3} J_{(\mathrm{H}, \mathrm{H})}=6.8,1 \mathrm{H}\right), 2.76(\mathrm{~d}$, $\left.{ }^{3} J_{(\mathrm{H}, \mathrm{H})}=6.8,6 \mathrm{H}\right) ;{ }^{13} \mathrm{C} \mathrm{NMR}\left(75 \mathrm{MHz}, \mathrm{CDCl}_{3}\right) \delta 178.2,61.4,33.5,29.8,19.1$ 


\section{$N$-Methoxy- $N$-methylpivalamide (2f)}

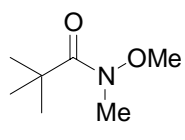

Compound $2 \mathbf{f}$ was synthesized from pivaloyl chloride and isolated as a yellow oil in $36 \%$ yield (UV purity: 93\%) after purification by flash chromatography (EtOAc/heptane 10:90): ${ }^{1} \mathrm{H}$ NMR and ${ }^{13} \mathrm{C}$ NMR spectral data were in agreement with those previously reported. ${ }^{5}$

$N$-Methoxy- $N$-methyl-4-nitrobenzamide (2h)

Compound $\mathbf{2 h}$ was synthesized from $p$-nitrobenzoyl chloride and isolated as yellow crystals in $85 \%$ yield (UV purity: $98 \%$ ) after recrystalization from ether: Mp $70-72{ }^{\circ} \mathrm{C}\left(\right.$ lit. $^{7} \mathrm{mp} 72-74{ }^{\circ} \mathrm{C}$ );

${ }^{1} \mathrm{H}$ NMR $\left(300 \mathrm{MHz}, \mathrm{CDCl}_{3}\right) \delta 8.26\left(\mathrm{~d},{ }^{3} J_{(\mathrm{H}, \mathrm{H})}=8.9,2 \mathrm{H}\right), 7.84\left(\mathrm{~d},{ }^{3} J_{(\mathrm{H}, \mathrm{H})}=8.9,2 \mathrm{H}\right), 3.52(\mathrm{~s}, 3$ H), $3.39(\mathrm{~s}, 3 \mathrm{H}) ;{ }^{13} \mathrm{C}$ NMR $\left(75 \mathrm{MHz}, \mathrm{CDCl}_{3}\right) \delta 167.6,148.8,140.0,129.2,123.2,61.3,33.1$; HRMS $(\mathrm{M}+\mathrm{H})^{+}$calcd. for $\mathrm{C}_{9} \mathrm{H}_{11} \mathrm{~N}_{2} \mathrm{O}_{4} 211.0719$, found 211.0701 .

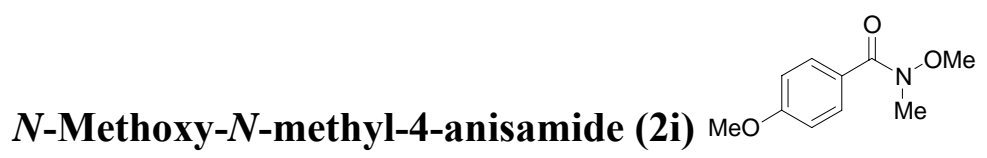

Compound $2 \mathbf{i}$ was synthesized from $p$-anisoyl chloride and isolated as a colorless oil in $63 \%$ yield (UV purity: 90\%) after purification by flash chromatography (EtOAc/heptane 10:90): ${ }^{1} \mathrm{H}$ NMR and ${ }^{13} \mathrm{C}$ NMR spectral data were in agreement with those previously reported. ${ }^{8}$

$N$-Methoxy- $N$-methyl-2,4-dimethoxybenzamide $(\mathbf{2} \mathbf{j}) \mathrm{MeO}_{\mathrm{Me}}$

Compound $\mathbf{2} \mathbf{j}$ was synthesized from 2,4-dimethoxybenzoyl chloride and isolated as a colorless solid in 73\% yield (UV purity: 92\%) after purification by flash chromatography (EtOAc/heptane 5:95 and then EtOAc/heptane 20:80): $\mathrm{Mp} 60-61{ }^{\circ} \mathrm{C} ;{ }^{1} \mathrm{H}$ NMR (300 MHz, $\left.\mathrm{CDCl}_{3}\right) \delta$ 7.24-7.21 (m, $1 \mathrm{H}) 6.51-6.47(\mathrm{~m}, 2 \mathrm{H}), 3.82(\mathrm{~s}, 3 \mathrm{H}), 3.59(\mathrm{~s}, 3 \mathrm{H}), 3.27(\mathrm{~s}, 3 \mathrm{H}) ;{ }^{13} \mathrm{C} \mathrm{NMR}\left(75 \mathrm{MHz}, \mathrm{CDCl}_{3}\right) \delta$ 
$167.9,161.7,157.2,128.8,117.6,104.3,98.5,60.8,55.5,55.3,33.4$; HRMS $(\mathrm{M}+\mathrm{H})^{+}$calcd. for $\mathrm{C}_{11} \mathrm{H}_{16} \mathrm{NO}_{4} 226.1079$, found 226.1053.

\section{$N$-Methoxy- $N$-methyl-2,4-Dichlorobenzamide (2k) c}

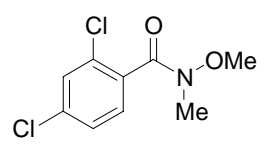

Compound $\mathbf{2 k}$ was synthesized from 2,4-dichlorobenzoyl chloride and isolated as a colorless oil in $81 \%$ yield (UV purity: $98 \%$ ) after purification by flash chromatography (EtOAc/hexane 10:90): ${ }^{1} \mathrm{H}$ NMR (300 MHz, $\left.\mathrm{CDCl}_{3}\right) \delta$ 7.43-7.28 (m, $\left.5 \mathrm{H}\right), 3.48(\mathrm{~s}, 3 \mathrm{H}), 3.35(\mathrm{~s}, 3 \mathrm{H}) ;{ }^{13} \mathrm{C} \mathrm{NMR}$ $\left(75 \mathrm{MHz}, \mathrm{CDCl}_{3}\right) \delta 167.4,135.6,133.7,131.8,129.5,128.6,126.9,61.4,32.3 ; \mathrm{HRMS}(\mathrm{M}+\mathrm{H})^{+}$ calcd. for $\mathrm{C}_{9} \mathrm{H}_{10} \mathrm{NO}_{2} \mathrm{Cl}_{2} 234.0089$, found 234.0075.

General procedure for the synthesis of $N$-methoxy- $N$-methyl- $\beta$-enaminoketoesters $3 \mathrm{a} / 4 \mathrm{a}$, $3 b / 4 b, 3 c / 4 c, 3 d / 4 d, 3 e / 4 e, 3 g, 3 h, 3 i$ and $3 k$

Under anhydrous conditions a 1.0 M THF-solution of sodium hexamethyldisilazide (2.0 mL, 2.0 mmol) was added to a THF-solution $(2.0 \mathrm{~mL})$ of ethyl propynoate $(2.0 \mathrm{mmol}, 205 \mu \mathrm{L})$ cooled at $78^{\circ} \mathrm{C}$. The addition was made dropwise during a period of 15 minutes followed by an additional half hour of stirring and addition of Weinreb amide $(1.0 \mathrm{mmol} 2)$. The mixture was heated to -40 ${ }^{\circ} \mathrm{C}$ during one hour and kept constant at that temp. until the reaction was quenched half an hour later by addition of $1 \mathrm{M}$ aq $\mathrm{HCl}(2 \mathrm{~mL})$. Stirring was continued while the mixture heated to ambient temp. Extraction with ether $(3 \times 25 \mathrm{~mL})$, drying $\left(\mathrm{MgSO}_{4}\right)$ of the comb. org. phases, filtration and evaporation to dryness by rotovap afforded the crude product $(\mathbf{3 a} / \mathbf{4 a}, \mathbf{3 b} / \mathbf{4} \mathbf{b}, \mathbf{3 c} / \mathbf{4} \mathbf{c}$, 3d/4d, 3e/4e, 3g, 3h, 3i and 3k). The products were isolated by flash chromatography (EtOAc/heptane 5:95) unless otherwise noted. 
( COOEt

and (E)-3-(N-methoxy- $N$-methyl-amino)-4-oxo-2-pentenoic acid ethyl ester (4a) $\mathrm{MeO}^{-{ }^{N}}{ }_{\mathrm{Me}}$

$N$-Methoxy- $N$-methylacetamide (2a, $106 \mu \mathrm{L}, 1.0 \mathrm{mmol})$ yielded 3a as colorless crystals (63 mg, 31\%): Mp 44-46 ${ }^{\circ} \mathrm{C} ;{ }^{1} \mathrm{H}$ NMR $\left(300 \mathrm{MHz}, \mathrm{CDCl}_{3}\right) \delta 5.32(\mathrm{~s}, 1 \mathrm{H}), 4.35\left(\mathrm{q},{ }^{3} J_{(\mathrm{H}, \mathrm{H})}=7.2,2 \mathrm{H}\right), 3.64$ $(\mathrm{s}, 3 \mathrm{H}), 3.01(\mathrm{~s}, 3 \mathrm{H}), 2.11(\mathrm{~s}, 3 \mathrm{H}), 1.34\left(\mathrm{t},{ }^{3} J_{(\mathrm{H}, \mathrm{H})}=7.2,3 \mathrm{H}\right) ;{ }^{13} \mathrm{C} \mathrm{NMR}\left(75 \mathrm{MHz}, \mathrm{CDCl}_{3}\right) \delta$ 194.6, 164.1, 152.4, 97.0, 62.2, 60.8, 37.5, 30.0, 13.7 and $\mathbf{4 a}$ as a colorless oil $(66 \mathrm{mg}, 33 \%):{ }^{1} \mathrm{H}$ NMR (300 MHz, $\left.\mathrm{CDCl}_{3}\right) \delta 4.71(\mathrm{~s}, 1 \mathrm{H}), 4.03\left(\mathrm{q},{ }^{3} J_{(\mathrm{H}, \mathrm{H})}=7.1,2 \mathrm{H}\right), 3.50(\mathrm{~s}, 3 \mathrm{H}), 2.90(\mathrm{~s}, 3 \mathrm{H})$, $2.36(\mathrm{~s}, 3 \mathrm{H}), 1.17\left(\mathrm{t},{ }^{3} J_{(\mathrm{H}, \mathrm{H})}=7.1,3 \mathrm{H}\right) ;{ }^{13} \mathrm{C} \mathrm{NMR}\left(75 \mathrm{MHz}, \mathrm{CDCl}_{3}\right) \delta 198.8,166.7,162.1,87.7$, 61.0, 59.7, 37.6, 30.3, 14.1. Purification by flash chromatography was performed with EtOAc/heptane 5:95 (4a) and then EtOAc/heptane 10:90 (3a) as eluent. The isolated crystals of 3a were used to define its structure by crystallography

(E)-2-(N-Methoxy- $N$-methyl-amino)-4-oxo-2-hexenoic acid ethyl ester (3b)

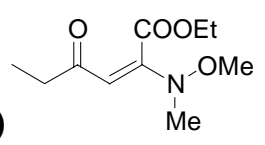

and $(E)-3-\left(N\right.$-methoxy- $N$-methyl-amino)-4-oxo-2-hexenoic acid ethyl ester (4b) $\mathrm{MeO}^{-N^{N}}{ }_{\mathrm{Me}}$

Compound $\mathbf{2 b}(117 \mathrm{mg}, 1.0 \mathrm{mmol})$ yielded $\mathbf{3 b}$ as a colorless oil $(88 \mathrm{mg}, 41 \%):{ }^{1} \mathrm{H}$ NMR (300 $\left.\mathrm{MHz}, \mathrm{CDCl}_{3}\right) \delta 5.29(\mathrm{~s}, 1 \mathrm{H}), 4.33\left(\mathrm{q},{ }^{3} J_{(\mathrm{H}, \mathrm{H})}=7.2,2 \mathrm{H}\right), 3.61(\mathrm{~s}, 3 \mathrm{H}), 2.97(\mathrm{~s}, 3 \mathrm{H}), 2.36(\mathrm{q}$, $\left.{ }^{3} J_{(\mathrm{H}, \mathrm{H})}=7.4,2 \mathrm{H}\right), 1.30\left(\mathrm{t},{ }^{3} J_{(\mathrm{H}, \mathrm{H})}=7.2,3 \mathrm{H}\right), 1.01\left(\mathrm{t},{ }^{3} J_{(\mathrm{H}, \mathrm{H})}=7.4,3 \mathrm{H}\right) ;{ }^{13} \mathrm{C} \mathrm{NMR}(75 \mathrm{MHz}$, $\left.\mathrm{CDCl}_{3}\right) \delta 197.7,164.1,152.1,96.6,62.0,60.7,37.5,35.8,13.7,8.3 ; \mathrm{HRMS}(\mathrm{M}+\mathrm{H})^{+}$calcd. for $\mathrm{C}_{10} \mathrm{H}_{18} \mathrm{NO}_{4}$ 216.1236, found 216.1217 and $\mathbf{4 b}$ as a colorless oil (73 mg, 34\%): ${ }^{1} \mathrm{H}$ NMR (300 $\left.\mathrm{MHz}, \mathrm{CDCl}_{3}\right) \delta 4.76(\mathrm{~s}, 1 \mathrm{H}), 4.05\left(\mathrm{q},{ }^{3} J_{(\mathrm{H}, \mathrm{H})}=7.1,2 \mathrm{H}\right), 3.52(\mathrm{~s}, 3 \mathrm{H}), 2.91(\mathrm{~s}, 3 \mathrm{H}), 2.67(\mathrm{q}$, $\left.{ }^{3} J_{(\mathrm{H}, \mathrm{H})}=7.2,2 \mathrm{H}\right), 1.19\left(\mathrm{t},{ }^{3} J_{(\mathrm{H}, \mathrm{H})}=7.1,3 \mathrm{H}\right), 1.14\left(\mathrm{t},{ }^{3} J_{(\mathrm{H}, \mathrm{H})}=7.2,3 \mathrm{H}\right) ;{ }^{13} \mathrm{C} \mathrm{NMR}(75 \mathrm{MHz}$, 
$\left.\mathrm{CDCl}_{3}\right) \delta 201.8,166.8,162.1,87.9,60.9,59.6,37.6,36.1,14.2,7.2$; HRMS $(\mathrm{M}+\mathrm{H})^{+}$calcd. for $\mathrm{C}_{10} \mathrm{H}_{18} \mathrm{NO}_{4} 216.1236$, found 216.1211. Purification by flash chromatography was performed with EtOAc/heptane 5:95 (4b) and then EtOAc/heptane 10:90 (3b) as eluent.

(E)-2-(N-Methoxy- $N$-methyl-amino)-4-oxo-5-phenyl-2-pentenoic acid ethyl ester

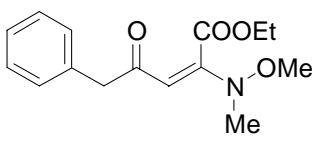

and $(E)-3-(N$-methoxy- $N$-methyl-amino)-4-oxo-5-phenyl-2-pentenoic

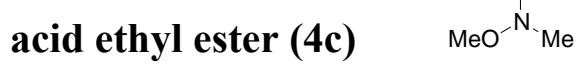

Compound $2 \mathbf{c}(179 \mathrm{mg}, 1.0 \mathrm{mmol})$ yielded $3 \mathbf{c}$ as a colorless oil (121 mg, 44\%): ${ }^{1} \mathrm{H}$ NMR (300 $\left.\mathrm{MHz}, \mathrm{CDCl}_{3}\right) \delta$ 7.21-7.12 (m, $\left.5 \mathrm{H}\right), 5.20(\mathrm{~s}, 1 \mathrm{H}), 4.29\left(\mathrm{q},{ }^{3} \mathrm{~J}_{(\mathrm{H}, \mathrm{H})}=7.1,2 \mathrm{H}\right), 3.57(\mathrm{~s}, 2 \mathrm{H}), 3.52$ (s, $3 \mathrm{H}), 2.90(\mathrm{~s}, 3 \mathrm{H}), 1.26\left(\mathrm{t},{ }^{3} J_{(\mathrm{H}, \mathrm{H})}=7.1,3 \mathrm{H}\right) ;{ }^{13} \mathrm{C} \mathrm{NMR}\left(75 \mathrm{MHz}, \mathrm{CDCl}_{3}\right) \delta$ 194.1, 163.9, 152.7, 135.3, 129.3, 128.4, 126.5, 95.7, 62.1, 60.6, 49.8, 37.3, 13.6; HRMS $(\mathrm{M}+\mathrm{H})^{+}$calcd. for $\mathrm{C}_{15} \mathrm{H}_{20} \mathrm{NO}_{4} 278.1392$, found 278.1400 and $4 \mathbf{c}$ as a colorless oil (82 $\left.\mathrm{mg}, 30 \%\right):{ }^{1} \mathrm{H}$ NMR (300 $\left.\mathrm{MHz}, \mathrm{CDCl}_{3}\right) \delta$ 7.33-7.26 (m, $\left.5 \mathrm{H}\right), 4.83(\mathrm{~s}, 1 \mathrm{H}), 4.14\left(\mathrm{q},{ }^{3} \mathrm{~J}_{(\mathrm{H}, \mathrm{H})}=7.1,2 \mathrm{H}\right), 4.03(\mathrm{~s}, 2 \mathrm{H}), 3.49$ $(\mathrm{s}, 3 \mathrm{H}), 2.85(\mathrm{~s}, 3 \mathrm{H}), 1.25\left(\mathrm{t},{ }^{3} J_{(\mathrm{H}, \mathrm{H})}=7.1,3 \mathrm{H}\right) ;{ }^{13} \mathrm{C} \mathrm{NMR}\left(75 \mathrm{MHz}, \mathrm{CDCl}_{3}\right) \delta$ 198.5, 166.9, $161.5,133.1,130.1,128.2,126.9,88.1,60.9,59.8,49.4,37.6,14.3$; HRMS $(\mathrm{M}+\mathrm{H})^{+}$calcd. for $\mathrm{C}_{15} \mathrm{H}_{20} \mathrm{NO}_{4} 278.1392$, found 278.1377. Purification by flash chromatography was performed with EtOAc/heptane 5:95 (4c) and then EtOAc/heptane 10:90 (3c) as eluent. 
(E)-2-(N-Methoxy- $N$-methyl-amino)-4-oxo-6-phenyl-2-hexenoic acid ethyl ester (3d) Me

and (E)-3-(N-methoxy- $N$-methyl-amino)-4-oxo-6-phenyl -2-hexenoic acid ethyl ester (4d)

Compound 2d (483 mg, $2.5 \mathrm{mmol})$ yielded 3d as a colorless oil (98 mg, 34\%): ${ }^{1} \mathrm{H}$ NMR (300 $\left.\mathrm{MHz}, \mathrm{CDCl}_{3}\right) \delta$ 7.29-7.17 (m, $\left.5 \mathrm{H}\right), 5.31(\mathrm{~s}, 1 \mathrm{H}), 4.40\left(\mathrm{q},{ }^{3} J_{(\mathrm{H}, \mathrm{H})}=7.2,2 \mathrm{H}\right), 3.65(\mathrm{~s}, 3 \mathrm{H}), 3.02$ $(\mathrm{s}, 3 \mathrm{H}), 2.92\left(\mathrm{t},{ }^{3} J_{(\mathrm{H}, \mathrm{H})}=7.8,2 \mathrm{H}\right), 2.71\left(\mathrm{t},{ }^{3} J_{(\mathrm{H}, \mathrm{H})}=8.2,2 \mathrm{H}\right), 1.34\left(\mathrm{t},{ }^{3} J_{(\mathrm{H}, \mathrm{H})}=7.2,3 \mathrm{H}\right) ;{ }^{13} \mathrm{C} \mathrm{NMR}$ $\left(75 \mathrm{MHz}, \mathrm{CDCl}_{3}\right) \delta 196.2,164.2,152.4,141.4,128.3,128.3,125.9,96.6,62.3,60.8,44.5,37.6$, 30.6, 13.8; HRMS $(M+H)^{+}$calcd. for $\mathrm{C}_{16} \mathrm{H}_{22} \mathrm{NO}_{4} 229.1549$, found 229.1522 and $4 \mathbf{d}$ as a colorless oil (96 mg, 33\%): ${ }^{1} \mathrm{H}$ NMR $\left(300 \mathrm{MHz}, \mathrm{CDCl}_{3}\right) \delta$ 7.31-7.18 (m, $\left.5 \mathrm{H}\right), 4.81$ (s, $\left.1 \mathrm{H}\right), 4.12$ $\left(\mathrm{q},{ }^{3} J_{(\mathrm{H}, \mathrm{H})}=7.1,2 \mathrm{H}\right), 3.48(\mathrm{~s}, 3 \mathrm{H}), 3.05(\mathrm{~s}, 4 \mathrm{H}), 2.91(\mathrm{~s}, 3 \mathrm{H}), 1.25\left(\mathrm{t},{ }^{3} J_{(\mathrm{H}, \mathrm{H})}=7.1,3 \mathrm{H}\right) ;{ }^{13} \mathrm{C}$ NMR $\left(75 \mathrm{MHz}, \mathrm{CDCl}_{3}\right) \delta 200.2,166.9,162.0,141.1,128.5,128.3,125.9,88.2,61.1,59.9,44.4$, 37.7, 29.2, 14.3; HRMS $(\mathrm{M}+\mathrm{H})^{+}$calcd. for $\mathrm{C}_{16} \mathrm{H}_{22} \mathrm{NO}_{4} 229.1549$, found 229.1563

(E)-2-(N-Methoxy- $N$-methyl-amino)-5-methyl-4-oxo-2-hexenoic acid ethyl ester (3e)<smiles>CCOC(=O)/C(=C\C(=O)C(C)C)N(C)OC</smiles>

and (E)-3-( $N$-methoxy- $N$-methyl-amino)-5-methyl-4-oxo-2-hexenoic acid ethyl ester (4e) $\mathrm{MeO}^{\prime} \mathrm{N}_{\mathrm{Me}}$

Compound $2 \mathrm{e}(131 \mathrm{mg}, 1.0 \mathrm{mmol})$ yielded $3 \mathbf{e}$ as a colorless oil $(120 \mathrm{mg}, 52 \%):{ }^{1} \mathrm{H}$ NMR (300 $\left.\mathrm{MHz}, \mathrm{CDCl}_{3}\right) \delta 5.32(\mathrm{~s}, 1 \mathrm{H}), 4.31\left(\mathrm{q},{ }^{3} J_{(\mathrm{H}, \mathrm{H})}=7.2,2 \mathrm{H}\right), 3.59(\mathrm{~s}, 3 \mathrm{H}), 2.97(\mathrm{~s}, 3 \mathrm{H}), 2.49$ (heptet, $\left.{ }^{3} J_{(\mathrm{H}, \mathrm{H})}=6.9,1 \mathrm{H}\right), 1.28\left(\mathrm{t},{ }^{3} J_{(\mathrm{H}, \mathrm{H})}=7.2,3 \mathrm{H}\right), 1.02\left(\mathrm{~d},{ }^{3} J_{(\mathrm{H}, \mathrm{H})}=6.9,6 \mathrm{H}\right) ;{ }^{13} \mathrm{C} \mathrm{NMR}(75 \mathrm{MHz}$, $\left.\mathrm{CDCl}_{3}\right) \delta 200.9,164.1,152.6,95.8,61.9,60.6,40.3,37.5,18.5,13.6$; HRMS $(\mathrm{M}+\mathrm{H})^{+}$calcd. for 
$\mathrm{C}_{11} \mathrm{H}_{20} \mathrm{NO}_{4}$ 230.1392, found 230.1366 and $4 \mathrm{e}$ as a colorless oil (83 mg, 36\%): ${ }^{1} \mathrm{H}$ NMR (300 $\left.\mathrm{MHz}, \mathrm{CDCl}_{3}\right) \delta 4.83(\mathrm{~s}, 1 \mathrm{H}), 4.04\left(\mathrm{q},{ }^{3} J_{(\mathrm{H}, \mathrm{H})}=7.1,2 \mathrm{H}\right), 3.52(\mathrm{~s}, 3 \mathrm{H}), 2.91(\mathrm{~s}, 3 \mathrm{H}), 2.83$ (heptet, $\left.{ }^{3} J_{(\mathrm{H}, \mathrm{H})}=7.0,1 \mathrm{H}\right), 1.19\left(\mathrm{t},{ }^{3} J_{(\mathrm{H}, \mathrm{H})}=7.1,3 \mathrm{H}\right), 1.17\left(\mathrm{~d},{ }^{3} J_{(\mathrm{H}, \mathrm{H})}=7.0,6 \mathrm{H}\right) ;{ }^{13} \mathrm{C} \mathrm{NMR}(75 \mathrm{MHz}$, $\left.\mathrm{CDCl}_{3}\right) \delta 205.2,166.9,161.1,88.4,60.5,59.6,40.8,37.8,17.5,14.2$; HRMS $(\mathrm{M}+\mathrm{H})^{+}$calcd. for $\mathrm{C}_{11} \mathrm{H}_{20} \mathrm{NO}_{4} 230.1392$, found 230.1344

(E)-2-(N-Methoxy- $N$-methyl-amino)-4-oxo-4-phenyl-2-butenoic acid ethyl ester (3g)

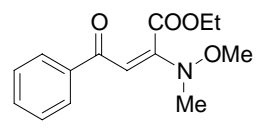

$N$-Methoxy- $N$-methylbenzamide $(\mathbf{2 g}, 155 \mu \mathrm{L}, 1.0 \mathrm{mmol}$ ) yielded $\mathbf{3 g}$ as a colorless oil (253 $\mathrm{mg}$, 96\%): ${ }^{1} \mathrm{H}$ NMR (300 MHz, $\left.\mathrm{CDCl}_{3}\right) \delta$ 7.92-7.88 (m, $\left.2 \mathrm{H}\right), 7.52-7.39$ (m, $\left.3 \mathrm{H}\right), 6.01$ (s, $\left.1 \mathrm{H}\right), 4.44$ $\left(\mathrm{q},{ }^{3} J_{(\mathrm{H}, \mathrm{H})}=7.2,2 \mathrm{H}\right), 3.72(\mathrm{~s}, 3 \mathrm{H}), 3.14(\mathrm{~s}, 3 \mathrm{H}), 1.38\left(\mathrm{t},{ }^{3} J_{(\mathrm{H}, \mathrm{H})}=7.2,3 \mathrm{H}\right) ;{ }^{13} \mathrm{C} \mathrm{NMR}(75 \mathrm{MHz}$, $\left.\mathrm{CDCl}_{3}\right) \delta 187.7,164.3,154.0,138.9,131.9,128.3,127.8,93.9,62.4,60.8,37.8,13.9 ;$ HRMS (M $+\mathrm{H})^{+}$calcd. for $\mathrm{C}_{14} \mathrm{H}_{18} \mathrm{NO} 4264.1236$, found 264.1261

(E)-2-( $N$-Methoxy- $N$-methyl-amino)-4-oxo-4-(4-nitrophenyl)-2-butenoic acid ethyl ester (3h)

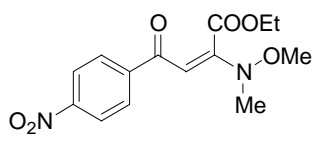

Compound $\mathbf{2 h}(210 \mathrm{mg}, 1.0 \mathrm{mmol})$ yielded $\mathbf{3 h}$ as a beige powder (231 $\mathrm{mg}, 75 \%)$ after washing the crude product with heptane: $\mathrm{Mp} 81-83{ }^{\circ} \mathrm{C} ;{ }^{1} \mathrm{H}$ NMR $\left(300 \mathrm{MHz}, \mathrm{CDCl}_{3}\right) \delta 8.26\left(\mathrm{~d},{ }^{3} \mathrm{~J}_{(\mathrm{H}, \mathrm{H})}=\right.$ 8.6, $2 \mathrm{H}), 8.03\left(\mathrm{~d},{ }^{3} J_{(\mathrm{H}, \mathrm{H})}=8.6,2 \mathrm{H}\right), 5.97(\mathrm{~s}, 1 \mathrm{H}), 4.47\left(\mathrm{q},{ }^{3} J_{(\mathrm{H}, \mathrm{H})}=7.1,2 \mathrm{H}\right), 3.76(\mathrm{~s}, 3 \mathrm{H}), 3.21$ $(\mathrm{s}, 3 \mathrm{H}), 1.41\left(\mathrm{t},{ }^{3} J_{(\mathrm{H}, \mathrm{H})}=7.1,3 \mathrm{H}\right) ;{ }^{13} \mathrm{C} \mathrm{NMR}\left(75 \mathrm{MHz}, \mathrm{CDCl}_{3}\right) \delta 185.4,163.6,154.5,149.5$, 144.2, 128.6, 123.5, 91.7, 62.7, 60.8, 37.5, 13.8; HRMS $(\mathrm{M}+\mathrm{H})^{+}$calcd. for $\mathrm{C}_{14} \mathrm{H}_{17} \mathrm{~N}_{2} \mathrm{O}_{6}$ 309.1087, found 309.1055 
(E)-2-(N-Methoxy- $N$-methyl-amino)-4-oxo-4-(4-anisoyl)-2-butenoic acid ethyl ester (3i)

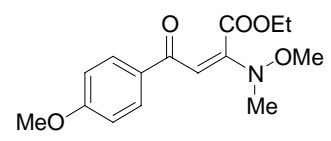

Compound $\mathbf{2 i}(195 \mathrm{mg}, 1.0 \mathrm{mmol})$ yielded $\mathbf{3 i}$ as an orange oil (210 $\mathrm{mg}, 72 \%$ ) after flash chromatography (EtOAc/heptane 10:90): ${ }^{1} \mathrm{H}$ NMR (300 MHz, $\left.\mathrm{CDCl}_{3}\right) \delta$ 7.87-7.83 (m, $2 \mathrm{H}$ ), 6.87-6.83 (m, $2 \mathrm{H}), 6.00(\mathrm{~s}, 1 \mathrm{H}), 4.38\left(\mathrm{q},{ }^{3} J_{(\mathrm{H}, \mathrm{H})}=7.1,2 \mathrm{H}\right), 3.77(\mathrm{~s}, 3 \mathrm{H}), 3.65(\mathrm{~s}, 3 \mathrm{H}), 3.06(\mathrm{~s}, 3$ $\mathrm{H}), 1.32\left(\mathrm{t},{ }^{3} J_{(\mathrm{H}, \mathrm{H})}=7.1,3 \mathrm{H}\right) ;{ }^{13} \mathrm{C} \mathrm{NMR}\left(75 \mathrm{MHz}, \mathrm{CDCl}_{3}\right) \delta 186.2,164.3,162.5,153.5,131.4$, 129.7, 113.2, 94.0, 61.9, 60.6, 55.1, 37.6, 13.6; HRMS $(\mathrm{M}+\mathrm{H})^{+}$calcd. for $\mathrm{C}_{15} \mathrm{H}_{20} \mathrm{NO}_{5}$ 294.1342, found 294.1313

(E)-2-( $N$-Methoxy- $N$-methyl-amino)-4-oxo-4-(2,4-dichlorophenyl)-2-butenoic acid ethyl

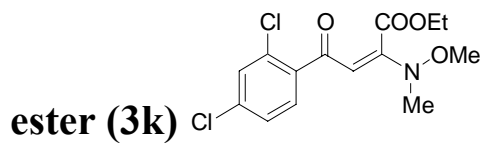

Compound 2k (234 mg, $1.0 \mathrm{mmol}$ ) yielded 3k as a white solid (199 mg, 60\%): $\mathrm{Mp} 72-73{ }^{\circ} \mathrm{C} ;{ }^{1} \mathrm{H}$ NMR (300 MHz, $\left.\mathrm{CDCl}_{3}\right) \delta$ 7.47-7.24 (m, $\left.3 \mathrm{H}\right), 5.70(\mathrm{~s}, 1 \mathrm{H}), 4.42\left(\mathrm{q},{ }^{3} J_{(\mathrm{H}, \mathrm{H})}=7.2,2 \mathrm{H}\right), 3.71(\mathrm{~s}$, $3 \mathrm{H}), 3.14(\mathrm{~s}, 3 \mathrm{H}), 1.38\left(\mathrm{t},{ }^{3} J_{(\mathrm{H}, \mathrm{H})}=7.2,3 \mathrm{H}\right) ;{ }^{13} \mathrm{C} \mathrm{NMR}\left(75 \mathrm{MHz}, \mathrm{CDCl}_{3}\right) \delta 187.4,163.6,153.2$, 138.8, 136.3, 132.0, 130.6, 129.9, 127.1, 96.2, 62.6, 60.9, 37.5, 13.8; HRMS $(\mathrm{M}+\mathrm{H})^{+}$calcd. for $\mathrm{C}_{14} \mathrm{H}_{16} \mathrm{NO}_{4} \mathrm{Cl}_{2} 332.0456$, found 332.0426

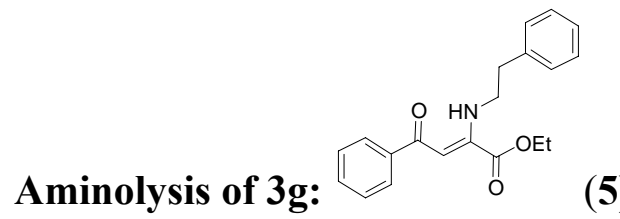

An EtOH-solution $(1 \mathrm{~mL})$ of $\mathbf{3 g}(26 \mathrm{mg}, 0.10 \mathrm{mmol})$ and phenethylamine $(14 \mu \mathrm{L}, 0.11 \mathrm{mmol})$ was stirred at ambient temperature for 13 hours. The solvent was then removed at reduced pressure by evaporation and the crude purified by flash chromatography (EtOAc/hexane 10:90). 5 
was isolated as a colorless oil (24 mg, 75\%): ${ }^{1} \mathrm{H}$ NMR (300 MHz, $\left.\mathrm{CDCl}_{3}\right) \delta 10.88(\mathrm{br} \mathrm{s}, 1 \mathrm{H})$, 7.90-7.87 (m, $2 \mathrm{H}), 7.50-7.21(\mathrm{~m}, 8 \mathrm{H}), 6.16(\mathrm{~s}, 1 \mathrm{H}), 4.40\left(\mathrm{q},{ }^{3} J_{(\mathrm{H}, \mathrm{H})}=7.1,2 \mathrm{H}\right), 3.72\left(\mathrm{dt},{ }^{3} J_{(\mathrm{H}, \mathrm{H})}=\right.$ 6.9, $2 \mathrm{H}), 2.95\left(\mathrm{t},{ }^{3} J_{(\mathrm{H}, \mathrm{H})}=7.4,2 \mathrm{H}\right), 1.34\left(\mathrm{t},{ }^{3} J_{(\mathrm{H}, \mathrm{H})}=7.1,3 \mathrm{H}\right) ;{ }^{13} \mathrm{C} \mathrm{NMR}\left(75 \mathrm{MHz}, \mathrm{CDCl}_{3}\right) \delta$ $190.4,163.5,153.2,139.5,138.2,131.4,128.9,128.6,128.3,127.2,126.6,92.4,62.1,46.7,37.6$, 14.1; HRMS $(\mathrm{M}+\mathrm{H})^{+}$calcd. for $\mathrm{C}_{20} \mathrm{H}_{22} \mathrm{NO}_{3} 324.1600$, found 324.1568

Reduction of 3g:

Compound $3 \mathrm{~g}(9.9 \mathrm{mg}, 0.030 \mathrm{mmol})$ was heated by microwaves at $150{ }^{\circ} \mathrm{C}$ in abs. EtOH $(0.6 \mathrm{~mL})$ for an hour. The product was isolated as a colorless oil (1.9 $\mathrm{mg}, 27 \%)$ by removal of the solvent at reduced pressure and purification by flash chromatography (EtOAc/heptane 10:90): ${ }^{1} \mathrm{H}$ NMR $\left(300 \mathrm{MHz}, \mathrm{CDCl}_{3}\right) \delta 10.74($ br s, $1 \mathrm{H}), 7.89-7.39(\mathrm{~m}, 5 \mathrm{H}), 6.19(\mathrm{~s}, 1 \mathrm{H}), 4.37\left(\mathrm{q},{ }^{3} J_{(\mathrm{H}, \mathrm{H})}=7.1,2\right.$ $\mathrm{H}), 3.14\left(\mathrm{~d},{ }^{3} J_{(\mathrm{H}, \mathrm{H})}=5.5,3 \mathrm{H}\right), 1.40\left(\mathrm{t},{ }^{3} J_{(\mathrm{H}, \mathrm{H})}=7.1,3 \mathrm{H}\right) ;{ }^{13} \mathrm{C} \mathrm{NMR}\left(75 \mathrm{MHz}, \mathrm{CDCl}_{3}\right) \delta 190.4$, $163.4,154.0,139.5,131.3,128.3,127.1,92.1,62.1,31.7,14.1$; HRMS $(\mathrm{M}+\mathrm{H})^{+}$calcd. for $\mathrm{C}_{13} \mathrm{H}_{16} \mathrm{NO}_{3} 234.1130$, found 234.1102

\section{General procedure for the synthesis of pyrazoles 7a-h}

A mixture of $N$-methoxy- $N$-methyl- $\beta$-enaminoketoester $\quad(\mathbf{3 a}, \quad \mathbf{d}, \quad \mathbf{g}$ and $\mathbf{k})$ and methyl/phenylhydrazine was heated by microwaves at $100{ }^{\circ} \mathrm{C}$ in dry $\mathrm{CDCl}_{3}(0.6 \mathrm{~mL})$ for $1(7 \mathbf{c}$, $7 \mathbf{d}, 7 \mathrm{e}, 7 \mathbf{g}$ and $7 \mathbf{h})$ or $2(\mathbf{7} \mathbf{a}, \mathbf{b}$, and $\mathbf{f})$ hours. The progression of the reaction was followed by ${ }^{1} \mathrm{H}$ NMR spectroscopy after one hour and completed by an additional hour of heating if the analysis was unsatisfactory. The product was isolated by removal of the solvent at reduced pressure by evaporation and purification by prep. HPLC. The physical data for $\mathbf{7 a}, \mathbf{7 b}, \mathbf{7 e}$ and $\mathbf{7 f}$ were in agreement with those previously reported. ${ }^{9}$ 


\section{1,5-Dimethyl-pyrazole-3-carboxylic acid ethyl ester (7a) O}

Compound 3a $(6.0 \mathrm{mg}, 0.030 \mathrm{mmol})$ and methylhydrazine $(2.0 \mu \mathrm{L}, 0.036 \mathrm{mmol})$ yielded $7 \mathbf{a}(2.1$ mg, 42\%, UV purity: 94\%) as colorless crystals: Mp 41-42 ${ }^{\circ} \mathrm{C}\left(\right.$ lit. $\left.^{10} \mathrm{mp} 42{ }^{\circ} \mathrm{C}\right)$.

\section{5-Methyl-1-phenyl-pyrazole-3-carboxylic acid ethyl ester (7b)}

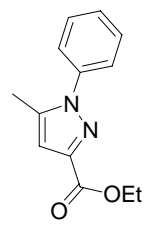

Compound 3a $(6.0 \mathrm{mg}, 0.030 \mathrm{mmol})$ and phenylhydrazine $(3.7 \mu \mathrm{L}, 0.036 \mathrm{mmol})$ yielded $7 \mathbf{b}(5.0$ mg, 72\%, UV purity: 93\%) as a white solid: Mp 31-32 ${ }^{\circ} \mathrm{C}\left(\right.$ lit. $\left.^{9} \mathrm{mp} 37{ }^{\circ} \mathrm{C}\right)$; HRMS $(\mathrm{M}+\mathrm{H})^{+}$ calcd. for $\mathrm{C}_{13} \mathrm{H}_{15} \mathrm{~N}_{2} \mathrm{O}_{2} 231.1134$, found 231.1155

\section{1-Methyl-5-phenylethyl-pyrazole-3-carboxylic acid ethyl ester (7c)}

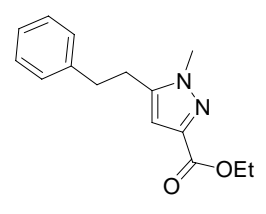

Compound 3d $(8.7 \mathrm{mg}, 0.030 \mathrm{mmol})$ and methylhydrazine $(2.0 \mu \mathrm{L}, 0.036 \mathrm{mmol})$ yielded $7 \mathbf{c}(4.6$ mg, 59\%, UV purity: 85\%) as a colorless oil: ${ }^{1} \mathrm{H} \mathrm{NMR}\left(300 \mathrm{MHz}, \mathrm{CDCl}_{3}\right) \delta 7.32-7.13(\mathrm{~m}, 5 \mathrm{H})$, $6.63(\mathrm{~s}, 1 \mathrm{H}), 4.41\left(\mathrm{q},{ }^{3} J_{(\mathrm{H}, \mathrm{H})}=7.1,2 \mathrm{H}\right), 3.68(\mathrm{~s}, 3 \mathrm{H}), 2.99-2.87(\mathrm{~m}, 4 \mathrm{H}), 1.25\left(\mathrm{t},{ }^{3} J_{(\mathrm{H}, \mathrm{H})}=7.1,3\right.$ $\mathrm{H}) ;{ }^{13} \mathrm{C}$ NMR $\left(75 \mathrm{MHz}, \mathrm{CDCl}_{3}\right) \delta 162.5,143.8,142.3,140.1,128.6,128.3,126.5,107.4,60.8$, 36.8, 34.9, 27.6, 14.4; HRMS $(\mathrm{M}+\mathrm{H})^{+}$calcd. for $\mathrm{C}_{15} \mathrm{H}_{19} \mathrm{~N}_{2} \mathrm{O}_{2} 259.1447$, found 259.1429

\section{1-Phenyl-5-phenylethyl-pyrazole-3-carboxylic acid ethyl ester (7d)}

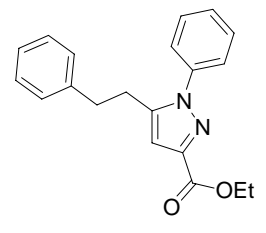

Compound 3d $(8.7 \mathrm{mg}, 0.030 \mathrm{mmol})$ and phenylhydrazine $(3.7 \mu \mathrm{L}, 0.036 \mathrm{mmol})$ yielded $7 \mathbf{d}(5.4$ mg, 54\%, UV purity: 94\%) as a white solid: $\mathrm{Mp} 64-66{ }^{\circ} \mathrm{C} ;{ }^{1} \mathrm{H} \mathrm{NMR}\left(300 \mathrm{MHz}, \mathrm{CDCl}_{3}\right) \delta 7.45-$ $7.04(\mathrm{~m}, 10 \mathrm{H}), 6.81(\mathrm{~s}, 1 \mathrm{H}), 4.42\left(\mathrm{q},{ }^{3} J_{(\mathrm{H}, \mathrm{H})}=7.1,2 \mathrm{H}\right), 2.96-2.87(\mathrm{~m}, 4 \mathrm{H}), 1.40\left(\mathrm{t},{ }^{3} J_{(\mathrm{H}, \mathrm{H})}=7.1\right.$, 
$3 \mathrm{H}) ;{ }^{13} \mathrm{C} \mathrm{NMR}\left(75 \mathrm{MHz}, \mathrm{CDCl}_{3}\right) \delta 162.6,144.7,143.9,140.1,139.0,129.1,128.8,128.5,128.2$, 126.4, 126.0, 108.0, 61.0, 34.9, 28.0, 14.1; HRMS $(\mathrm{M}+\mathrm{H})^{+}$calcd. for $\mathrm{C}_{20} \mathrm{H}_{21} \mathrm{~N}_{2} \mathrm{O}_{2}$ 321.1603, found 321.1586

1-Methyl-5-phenyl-pyrazole-3-carboxylic acid ethyl ester (7e)

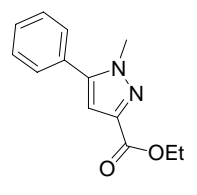

Compound $3 \mathrm{~g}$ ( $7.9 \mathrm{mg}, 0.030 \mathrm{mmol})$ and methylhydrazine $(2.0 \mu \mathrm{L}, 0.036 \mathrm{mmol})$ yielded $7 \mathrm{e}(15.9$ mg, 69\%, UV purity: 95\%) as a colorless oil: HRMS $(\mathrm{M}+\mathrm{H})^{+}$calcd. for $\mathrm{C}_{13} \mathrm{H}_{15} \mathrm{~N}_{2} \mathrm{O}_{2} 231.1134$, found 231.1125

\section{1,5-Diphenyl-pyrazole-3-carboxylic acid ethyl ester (7f)}

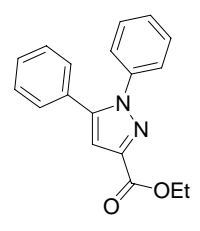

Compound $3 \mathbf{g}$ ( $7.9 \mathrm{mg}, 0.030 \mathrm{mmol})$ and phenylhydrazine $(3.7 \mu \mathrm{L}, 0.036 \mathrm{mmol})$ yielded $7 \mathbf{f}(8.2$ mg, 94\%, UV purity: $90 \%$ ) as a yellow solid: Mp 58-60 ${ }^{\circ} \mathrm{C}\left(\right.$ lit. $\left.{ }^{9} \mathrm{mp} 56-58^{\circ} \mathrm{C}\right)$; HRMS $(\mathrm{M}+\mathrm{H})^{+}$ calcd. for $\mathrm{C}_{18} \mathrm{H}_{17} \mathrm{~N}_{2} \mathrm{O}_{2} 293.1290$, found 293.1270

\section{1-Methyl-5-(2, 4-dichlorophenyl)-pyrazole-3-carboxylic acid ethyl ester (7g)}

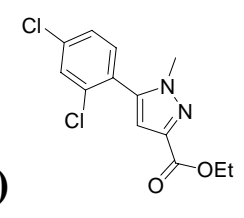

Compound 3k $(9.9 \mathrm{mg}, 0.030 \mathrm{mmol})$ and methylhydrazine $(2.0 \mu \mathrm{L}, 0.036 \mathrm{mmol})$ yielded $\mathbf{7 g}(6.8$ $\mathrm{mg}, 76 \%$, UV purity: $98 \%$ ) as a white solid: $\mathrm{Mp} 81-82{ }^{\circ} \mathrm{C} ;{ }^{1} \mathrm{H} \mathrm{NMR}\left(300 \mathrm{MHz}, \mathrm{CDCl}_{3}\right) \delta 7.55-$ $7.25(\mathrm{~m}, 3 \mathrm{H}), 6.82(\mathrm{~s}, 1 \mathrm{H}), 4.42\left(\mathrm{q},{ }^{3} J_{(\mathrm{H}, \mathrm{H})}=7.1,2 \mathrm{H}\right), 3.78(\mathrm{~s}, 3 \mathrm{H}), 1.41\left(\mathrm{t},{ }^{3} J_{(\mathrm{H}, \mathrm{H})}=7.1,3 \mathrm{H}\right)$; ${ }^{13} \mathrm{C}$ NMR $\left(75 \mathrm{MHz}, \mathrm{CDCl}_{3}\right) \delta 162.1,142.8,140.8,136.5,135.2,132.6,130.0,127.5,127.4$, 110.2, 61.0, 37.8, 14.4; HRMS $(\mathrm{M}+\mathrm{H})^{+}$calcd. for $\mathrm{C}_{13} \mathrm{H}_{13} \mathrm{~N}_{2} \mathrm{O}_{2} \mathrm{Cl}_{2} 299.0354$, found 299.0338 
1-Phenyl-(2, 4-dichlorophenyl)-pyrazole-3-carboxylic acid ethyl ester (7h)

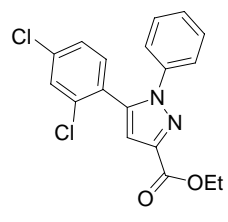

Compound 3k $(9.9 \mathrm{mg}, 0.030 \mathrm{mmol})$ and phenylhydrazine $(3.7 \mu \mathrm{L}, 0.036 \mathrm{mmol})$ yielded $7 \mathbf{h}(7.2$ mg, 67\%, UV purity: 98\%) as a brown solid: $\mathrm{Mp} 29-31{ }^{\circ} \mathrm{C} ;{ }^{1} \mathrm{H}$ NMR $\left(300 \mathrm{MHz}, \mathrm{CDCl}_{3}\right) \delta 7.43-$ $7.15(\mathrm{~m}, 8 \mathrm{H}), 7.04(\mathrm{~s}, 1 \mathrm{H}), 4.46\left(\mathrm{q},{ }^{3} J_{(\mathrm{H}, \mathrm{H})}=7.1,2 \mathrm{H}\right), 1.43\left(\mathrm{t},{ }^{3} J_{(\mathrm{H}, \mathrm{H})}=7.1,3 \mathrm{H}\right) ;{ }^{13} \mathrm{C}$ NMR $(75$ $\left.\mathrm{MHz}, \mathrm{CDCl}_{3}\right) \delta 162.2,144.3,140.2,139.2,136.1,134.8,132.7,130.0,129.0,128.3,127.8$, 127.3, 124.7, 111.9, 61.2, 14.4; HRMS $(\mathrm{M}+\mathrm{H})^{+}$calcd. for $\mathrm{C}_{18} \mathrm{H}_{15} \mathrm{~N}_{2} \mathrm{O}_{2} \mathrm{Cl}_{2}$ 361.0511, found 361.0481

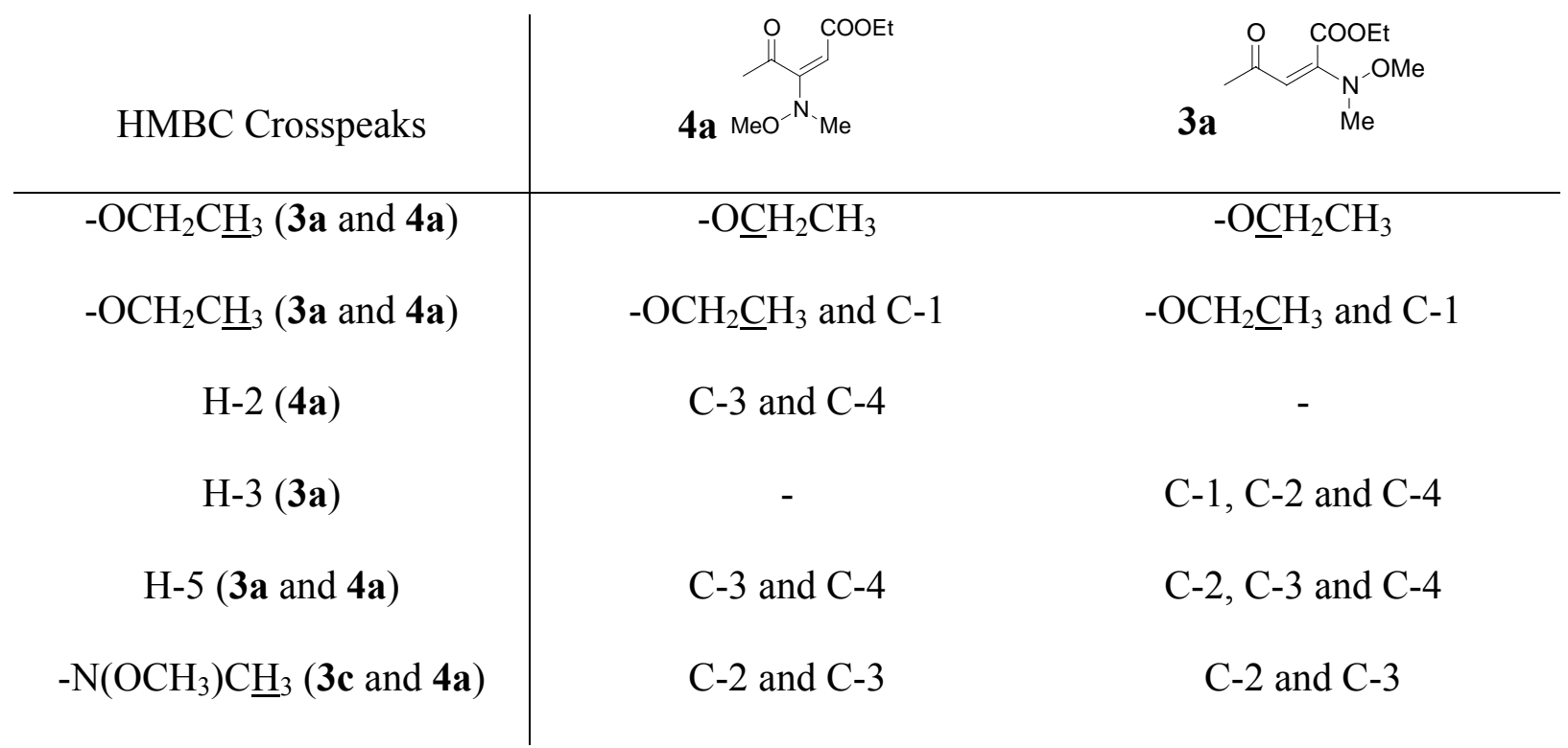




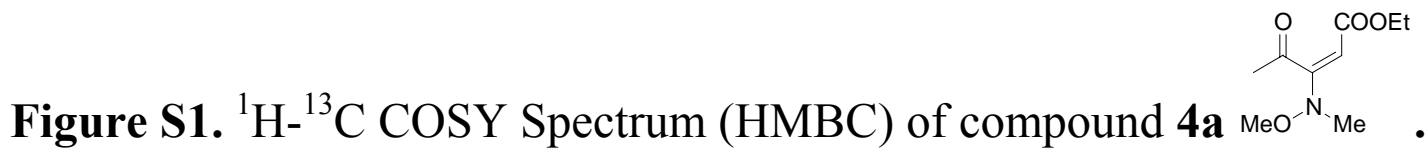
,

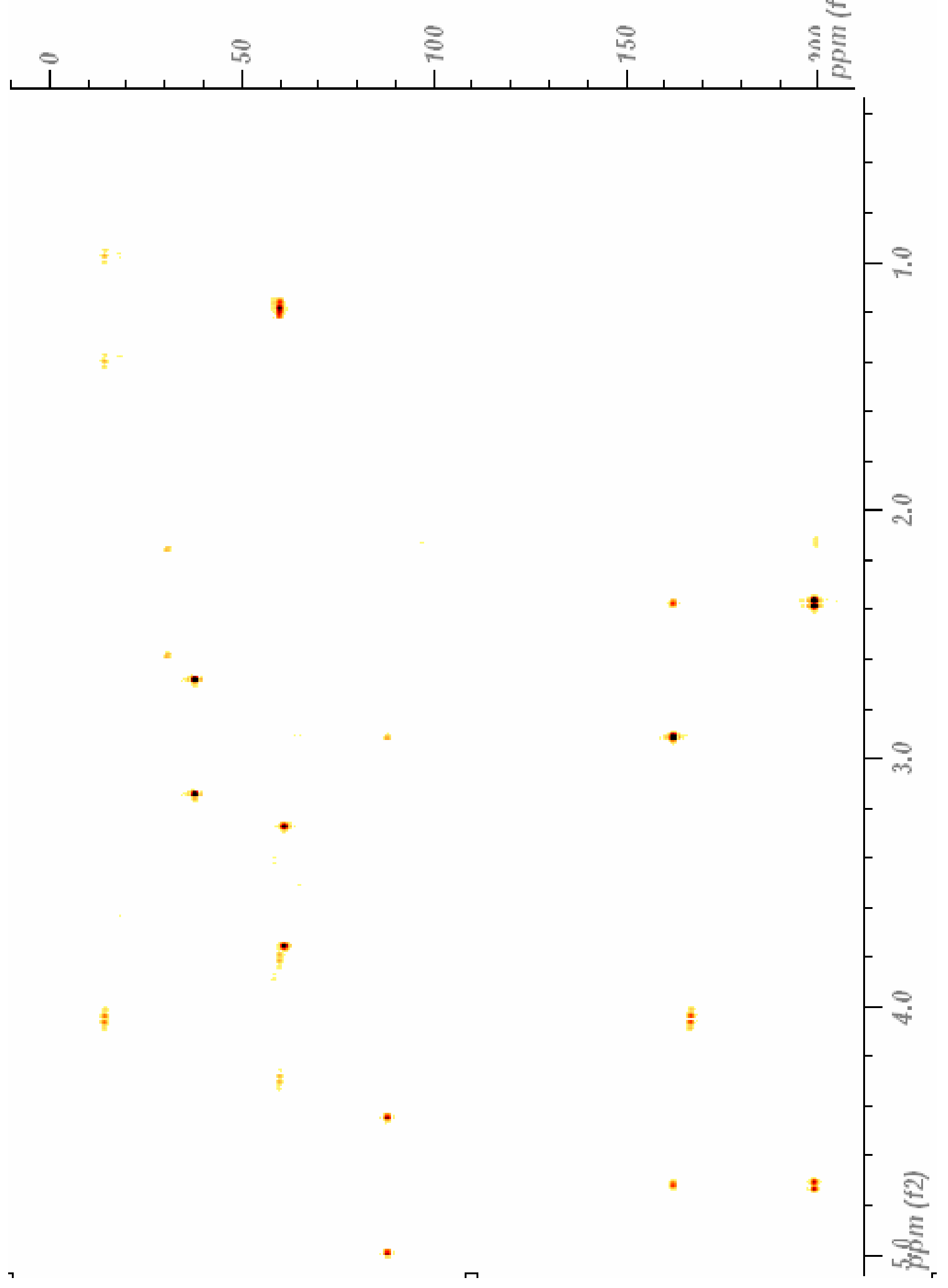


Figure S2. ${ }^{1} \mathrm{H}-{ }^{1} \mathrm{H}$ COSY (NOESY) Spectrum of compound 4a мео ${ }^{-{ }^{N}}$ ме

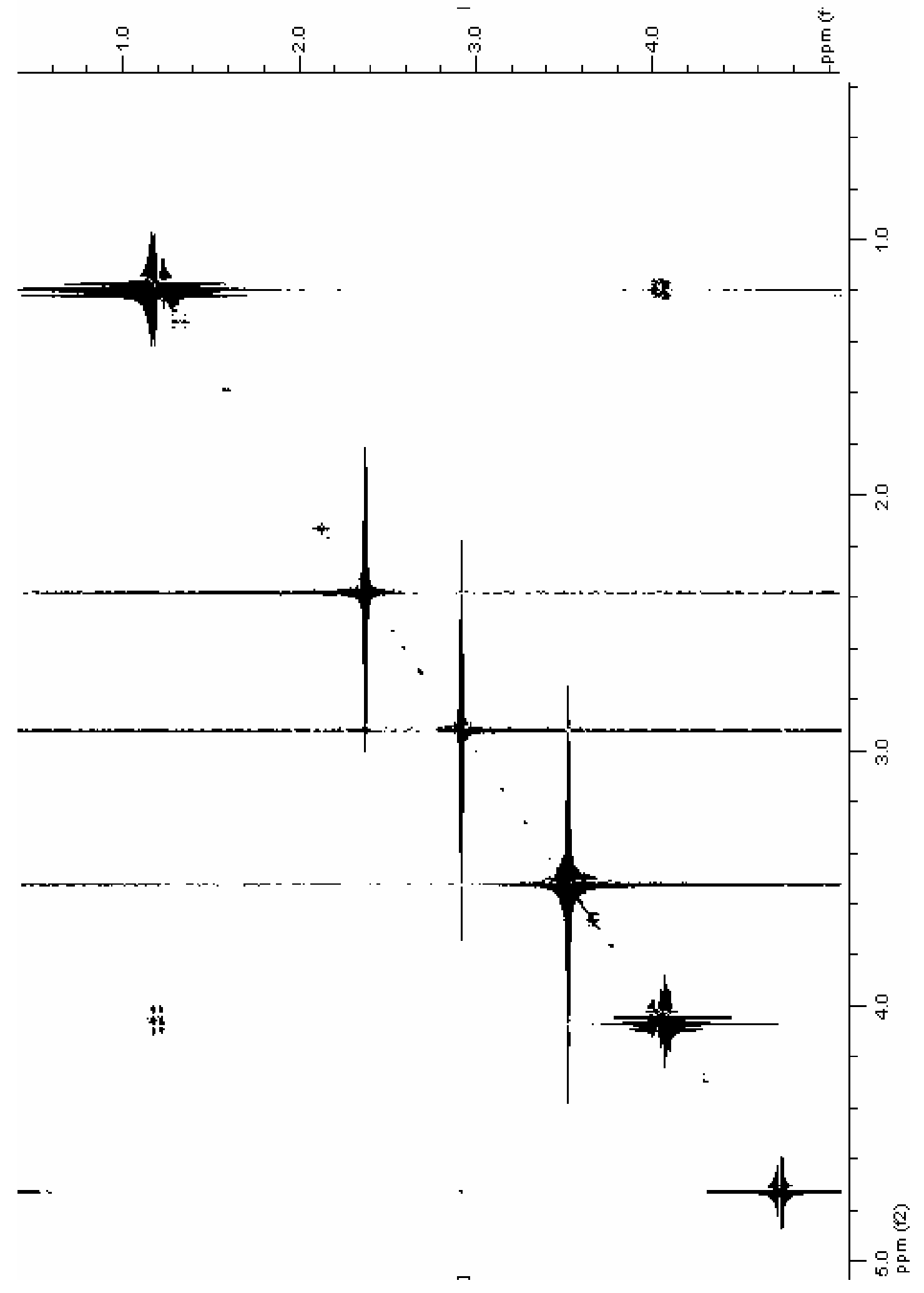


Figure S3. ${ }^{1} \mathrm{H}-{ }^{13} \mathrm{C}$ COSY Spectrum (HMBC) of compound $\mathbf{4 b}$
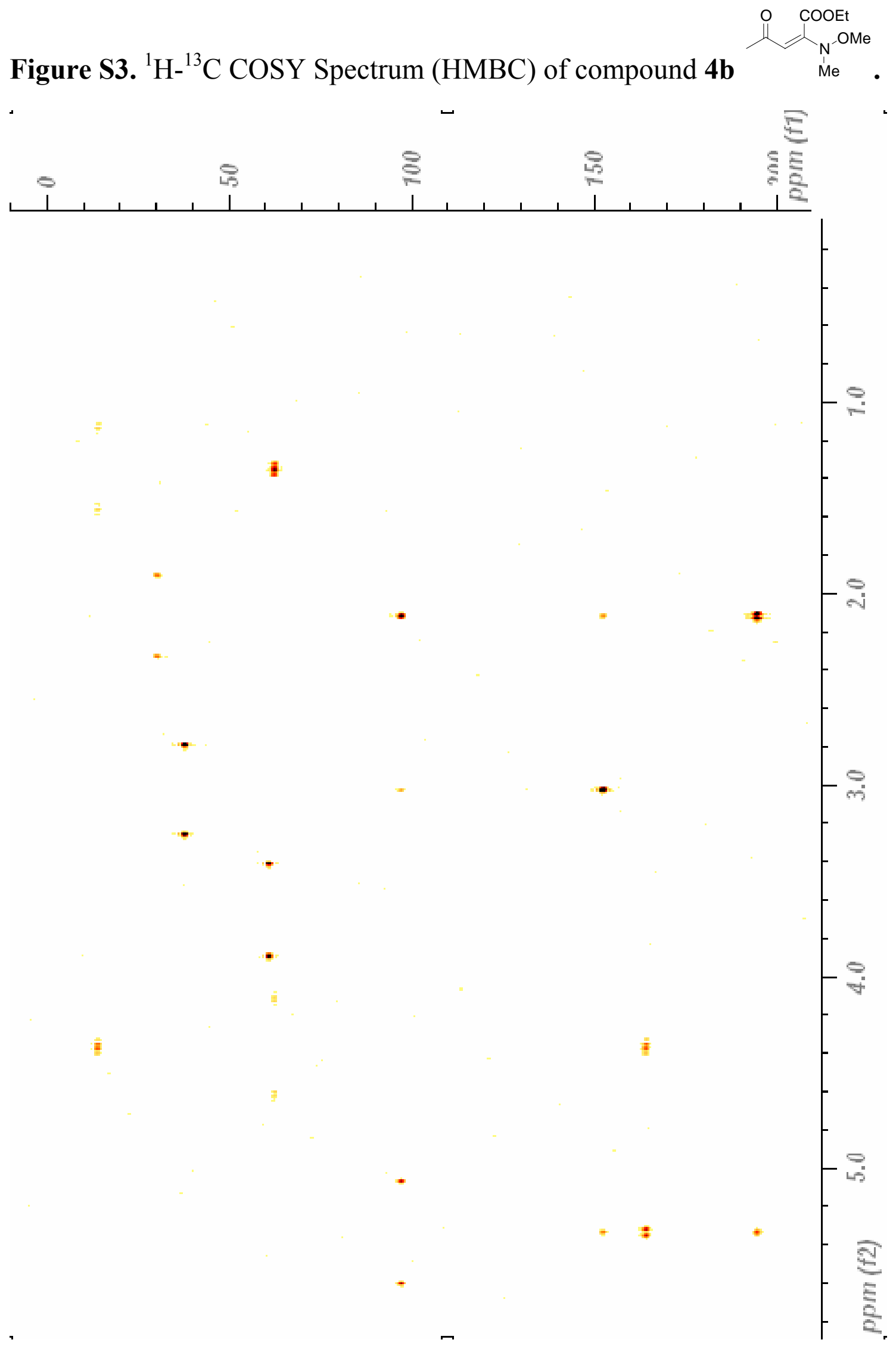


\section{References}

(1) Nikolai, J.; Maas, G. Synthesis 2003, 17, 2679-2688.

(2) Yerino, L. V.; Osborn, M. E.; Marian, P. S. Tetrahedron 1982, 38, 1579-1591.

(3) Li, X.; Hewgley, J. B.; Mulrooney, C. A.; Yang, J.; Kozlowski, M. C. J. Org. Chem. 2003, $68,5500-5511$.

(4) Wolberg, M.; Hummel, W.; Müller, M. Chem. Eur. J. 2001, 7, 4562-4571.

(5) Banwell, M.; Smith, J. Synth. Comm. 2001, 31, 2011-2019.

(6) Murphy, J. A.; Commeureuc, A. G. J.; Snaddon, T. N.; McGuire, T. M.; Khan, T. A.; Hisler, K.; Dewis, M. L.; Carling, R. Org. Lett. 2005, 7, 1427-1429.

(7) Beart, P. M.; Ward, A. D. Aust. J. Chem. 1974, 27, 1341-1349.

(8) Sofikiti, N.; Tofi, M.; Montagnon, T.; Vassilikogiannakis, G.; Stratakis, M. Org. Lett. 2005, 7, 2357-2359.

(9) Schmidt, A.; Habeck, T.; Kindermann, M. K.; Nieger, M. J. Org. Chem. 2003, 68, 5977-5982.

(10) Mühlstädt, v. M.; Zschiedrich, J. J. Prakt. Chem. 1969, 311, 363-369. 